1177-1182 (1995)

7. Platt, J.L. et al. Transplantation 52, 214-220 (1991).

8. Thall, A.D., Malý, P. \& Lowe, J.B. J. Biol. Chem. 270 21437-21440 (1995)

9. Huber-Lang, M. et al. J. Immunol. 166, 1193-1199 (2001).

\section{European agbiotech crisis?}

To the editor:

The ongoing political controversy surrounding plant biotechnology in Europe is having serious negative consequences not only for the industry, but also for the conduct of plant biology. Modern plant biology involves the routine creation of genetically altered lines that are grown up for scientific evaluation. The impact of many genes on plant phenotype can only be evaluated under field conditions that are difficult to duplicate in the laboratory. However, the lack of political support for agricultural biotechnology in Europe has strongly discouraged such studies, as opposed to merely inhibiting the development of new products. Here, we present an analysis of publicly available data that documents this disturbing trend (Table 1).

The dramatic fall in the number of applications for field trials since 1997 might be attributed to a decline in commercial interest in plant biotechnology.
However, inspection of a subset of these data for trees only, yields a different conclusion. Approximately $80 \%$ of the applications for the release of genetically modified (GM) trees do not involve a commercial organization, and all seem remote from any deployment. Thus, the decline in applications for field trials appears to reflect a real impact on the conduct of research to evaluate biotic and abiotic environmental effects on trees. The negative effects of this trend on woody plant biology in Europe will continue to be felt for many years to come.

The chilly political climate for plant biotechnology has often been blamed for the loss of commercial interest in plants, and the declining confidence of scientists in this discipline. To this list must also be added circumscription of the actual scientific enterprise.

Trevor M. Fenning and Jonathan Gershenzon, Max-Planck-Institut für Chemische Ökologie, Plant Biochemistry and Molecular Biology Department, Beutenberg Campus, Winzerlaer Str. 10 D-07745 Jena, Germany, (fenning@ice.mpg.de)
Table 1. The rise and fall of GM field trials within the EU

\begin{tabular}{lll} 
Year & All plants & Trees only \\
\hline 1990 & 0 & 0 \\
1991 & 4 & 0 \\
1992 & 66 & 0 \\
1993 & 89 & 2 \\
1994 & 166 & 1 \\
1995 & 213 & 5 \\
1996 & 239 & 6 \\
1997 & 264 & 3 \\
1998 & 244 & 7 \\
1999 & 238 & 4 \\
2000 & 129 & 4 \\
2001 & 88 & 2 \\
2002 & 35 & 1 \\
$1 Q 2003$ & 21 & 2 \\
Total & 1796 & 37 \\
\hline
\end{tabular}

1These data was taken from the web site for the Joint Research Centre (JRC) of the EU (http://engl.jrc.it/), which records all applications for the deliberate release of genetically modified (GM) organisms, other than permitted commercial deployments. The data on the JRC website were last verified on 14 March 2003.

${ }^{2}$ This is a subset of the applications for all plants. Each application can be for more than one GM plant line and/or species, however, and some trials with trees are still ongoing from previous years. The numbers for the GM traits and species tested show a similar pattern to that for the applications, but peaks at 11 in 1996 and declines to only one in 2002. The total number of GM traits and tree species tested to date is 47 . 